\title{
TUNING OF FUZZY SHARPENING FILTERS FOR BIOMEDICAL IMAGE ENHANCEMENT
}

\author{
Jaromir Kukal $^{1 *}$, Abduljalil Sireis ${ }^{2}$, Zuzana Krbcova ${ }^{2}$ \\ ${ }^{1}$ Czech Technical University in Prague \\ Faculty of Nuclear Sciences and Physical Engineering \\ Department of Software Engineering \\ Trojanova 13, 12000 Prague 2 \\ Czech Republic \\ jaromir.kukal@fjfi.cvut.cz \\ ${ }^{2}$ University of Chemistry and Technology in Prague \\ Faculty of Chemical Engineering \\ Department of Computing and Control Engineering \\ Technicka 5, 16626 Prague 6 \\ Czech Republic
}

\begin{abstract}
Various approaches are used for image smoothing and sharpening. The class of fuzzy filters is widely used in the case of spiky noise due to their non-linear behavior. A lot of popular fuzzy filters are realizable in Eukasiewicz algebra with square root. Frequently applied low-pass fuzzy filters were selected from literature and used for the image sharpening with dyadic weights. The first aim of the paper is to find the optimum sharpening with the best Signal-to-Noise Ratio criterion for various noise types and offer general suggestions for fuzzy filter selection. Our results are directly applicable to tomographic images from MRI, PET and SPECT scanners.
\end{abstract}

Keywords: image smoothing, image sharpening, Lukasiewicz algebra, fuzzy image processing, filter bank, MRI, PET, SPECT

\section{Introduction}

There are many approaches for designing linear and nonlinear 2D image enhancement. As typical for biomedical SPECT, PET, and MRI scans, the spiky noise has to be eliminated by statistically robust filters. The paper is based on fuzzy image processing in Lukasiewicz algebra with square root $[1,2]$ which enables to realize robust low-pass and sharpening filters. The enhancement quality is measured by Signal to Noise Ratio (SNR) and various combinations of integer masks and sharpening approaches are applied to biomedical image with Gaussian and spiky noise. There is only a finite number of combinations and the most suitable ones are discussed.

Lukasiewicz algebra with square root is remembered in the second section. The next section summarizes several operations which are useful for data processing. The frame of fuzzy local image processing and computer experiment design are established in the fourth section. The fifth section summarizes the main results of optimal sharpening with the highest possible SNR [3] which is followed by concluding remarks.

\section{2 Łukasiewicz Algebra with Square Root}

The main idea behind the scope of the paper is to compare real properties of various fuzzy filters. First, it is necessary to specify the collection of permitted operations. Supposing intensity normalization into [0, 1] interval, we decide to apply Many-Valued Algebra (MVA) [4]. Let $\mathcal{L}=[0,1]$ be supporting set. The Eukasiewicz algebra with square root [2] is defined as

$$
\mathrm{LA}_{\mathrm{sqrt}}=\langle\mathcal{L}, \wedge, \vee, \otimes, \rightarrow, 0,1, \text { sqrt }\rangle
$$

where

- $a \wedge b=\min (a, b)$,

- $a \vee b=\max (a, b)$,

- $0=\min \mathcal{L}$, 
- $1=\max \mathcal{L}$

- $a \otimes b=\max (a+b-1,0)$,

- $a \rightarrow b=\min (1-a+b, 1)$,

- $\operatorname{sqrt}(a)=\sqrt{a}=(1+a) / 2$.

Derived operators of $\mathrm{LA}_{\text {sqrt }}$ are

- $\neg a=1-a$,

- $a \oplus b=\neg(\neg a \otimes \neg b)=\min (a+b, 1)$,

- $a \ominus b=a \otimes \neg b=\max (a-b, 0)$.

Given algebra is frequently used in fuzzy image processing [5].

\section{Permitted Operations in $\mathrm{LA}_{\text {sqrt }}$}

The main question is how to design selected operations for signal and image processing in given algebra. Fortunately, $\mathrm{LA}_{\mathrm{sqrt}}$ is able to realize both traditional operators [5] and constrainded linear transforms with dyadic weights. Let $a, b, c \in[0,1]$ be fuzzy variables, $k$ be order in sorted list of $n$ values, $H \in \mathbb{N}_{0}$ be dyadic exponent, and $\alpha=m / 2^{N}$ be dyadic weight. There are several cases:

- When $a+b \leq 1$, standard addition is realizable as $a+b=a \oplus b$.

- When $a \geq b$, standard subtraction is realizable as $a-b=a \ominus b$.

- Using functions $\varphi(x)=\operatorname{sqrt}(x) \ominus(1 / 2)=x / 2$ and $\operatorname{trim}(x)=\min (1, \max (0, x))$ we can realize operations based on traditional multiplication by dyadic weight.

The last case can be proven as follows. First, $x / 2^{H}=\varphi^{[H]}(x)$ where $\varphi^{[0]}(x)=x$, $\varphi^{[H]}(x)=\varphi\left(\varphi^{[H-1]}(x)\right)$. Therefore, for $m x \leq 2^{N}$ we directly have

$$
\frac{m \cdot x}{2^{H}}=\bigoplus_{k=1}^{m} \varphi^{[H]}(x)
$$

which will be useful for realization of several linear filters.

Traditional image sharpening is unconstrained and based on formula $a+\alpha \cdot(b-c)$ which has to be trimmed into $[0,1]$ interval in the case of fuzzy processing. Therefore, we directly calculate

$$
\operatorname{trim}(a+\alpha \cdot(b-c))=a \oplus \alpha \cdot(b \ominus c) \ominus \alpha \cdot(c \ominus b) .
$$

Finally, when the data list of $n$ values is sorted as

$$
x_{(1)} \leq x_{(2)} \leq \ldots \leq x_{(n)}
$$

the $k^{\text {th }}$ value $x_{(k)}$ can be expressed in Disjoint Normal Form (DNF) and directly calculated using operators $\vee, \wedge$.

\section{Fuzzy Local Image Processing}

Using simple tricks from previous section, we can perform selected procedures of local image processing. Basic terms and operations are recalled first. Let $M, N \in \mathbb{N}$ be height and width of normalized image $\mathbf{X} \in[0,1]^{M \times N}$. The local image processing operates on list of values [6] from pixel neighborhood. The values are represented by vector $\mathbf{x} \in[0,1]^{m}$ of length $m \in \mathbb{N}$. It can be connected with vector $\mathbf{y} \in[0,1]^{n}$ of length $n \in \mathbb{N}$ using operator

$$
\mathbf{x} \sqcup \mathbf{y}=\left(x_{1}, x_{2}, \ldots, x_{m}, y_{1}, y_{2}, \ldots, y_{n}\right)^{\mathrm{T}} .
$$

Aggregation operator [3] is therefore defined as

$$
\bigsqcup_{k=1}^{N} \mathbf{x}_{k}=\mathbf{x}_{1} \sqcup \mathbf{x}_{2} \sqcup \ldots \sqcup \mathbf{x}_{n} .
$$


Multiple evidence of values can be obtained by multiplication operator

$$
n \odot \mathbf{x}=\bigsqcup_{k=1}^{N} \mathbf{x}
$$

which will be also useful for mask applications.

The integer mask of radius $r \in \mathbb{N}$ is represented by matrix $\mathbf{W} \in \mathbb{N}_{0}{ }^{(2 r+1) \times(2 r+1)}$ with central symmetry. The local processing with mask $\mathbf{W}$ of image part $\mathbf{B} \in[0,1]^{(2 r+1) \times(2 r+1)}$ generates the weighted list of values [3] as vector

$$
\mathbf{x}=\bigsqcup_{i=1}^{2 r+1} \bigsqcup_{j=1}^{2 r+1}\left(w_{i, j} \odot b_{i, j}\right)
$$

of length $n$ and any permitted operation from $\mathrm{LA}_{\text {sqrt }}$ can be used for local processing.

The local fuzzy image processing is frequently based on the evaluation of sample statistics:

- Erosion [3] as $\mathrm{E}(\mathbf{x})=\bigwedge_{k=1}^{n} x_{k}$,

- Dilation [3] as $\mathrm{D}(\mathbf{x})=\bigvee_{k=1}^{n} x_{k}$,

- Median [7, 8] for odd $n$ as $\mathrm{M}(\mathbf{x})=x_{(k)}$ where $k=(n+1) / 2$,

- Median for even $n$ as $\left.\mathrm{M}(\mathbf{x})=\varphi\left(x_{(k)}\right) \oplus \varphi x_{(k+1)}\right)$ where $k=n / 2$,

- First quartile [9] as $\mathrm{Q}_{1}(\mathbf{x})=\mathrm{M}\left(x_{(1)}, \ldots, x_{(k)}\right)$ where $k=\lfloor n / 2\rfloor$,

- Third quartile [9] as $\mathrm{Q}_{3}(\mathbf{x})=\mathrm{M}\left(x_{(k)}, \ldots, x_{(n)}\right)$ where $k=\lceil n / 2\rceil+1$,

- Average [10] for dyadic $n=2^{H}$ as $\mathrm{A}(\mathbf{x})=\bigoplus_{k=1}^{n} \varphi^{[H]}\left(x_{(k)}\right)$.

More sophisticated statistical calculations are based on Walsh list [11] which was originally published as

$$
\mathrm{W}(\mathbf{x})=\bigsqcup_{i \leq j} \frac{x_{i}+x_{j}}{2}
$$

but can be expressed in fuzzy form

$$
\mathrm{W}(\mathbf{x})=\bigsqcup_{i=1}^{n} \bigsqcup_{j=i}^{n}\left(\varphi\left(x_{i}\right) \oplus \varphi\left(x_{j}\right)\right)
$$

The other useful local operators are [3]:

- Hodges-Lehmann Median [11] as $\mathrm{H}(\mathbf{x})=\mathrm{M}(\mathrm{W}(\mathbf{x}))$,

- Opening $\mathrm{O}(\mathbf{x})$ as erosion followed by dilation,

- Closing $\mathrm{C}(\mathbf{x})$ as dilation followed be erosion,

- $O C$ Mean $\operatorname{OCM}(\mathbf{x})=\varphi(\mathrm{O}(\mathbf{x})) \oplus \varphi(\mathrm{C}(\mathbf{x}))$,

- $O C$ Filter $\mathrm{OC}(\mathbf{x})$ as opening followed by closing,

- CO Filter $\mathrm{CO}(\mathbf{x})$ as closing followed by opening,

- $O C C O \mathrm{Mean}$ $\operatorname{OCCOM}(\mathbf{x})=\varphi(\mathrm{OC}(\mathbf{x})) \oplus \varphi(\mathrm{CO}(\mathbf{x}))$,

- Constrained Filter $\mathrm{CF}(\mathbf{x})=\left(\mathrm{A}(\mathbf{x}) \vee \mathrm{Q}_{1}(\mathbf{x})\right) \wedge \mathrm{Q}_{3}(\mathbf{x})$ and the central weight of mask $\mathbf{W}$ needs to be replaced by zero. 
Previous approaches can be used for Fuzzy Low-Pass (FLP) [12] filtering which should improve the image quality. Another possibility is to apply FLP filter in image sharpening process using $x_{\mathrm{c}}$ as intensity behind the mask center. Resulted sharpening operator

$$
\mathrm{S}(\mathbf{x})=\mathrm{FLP}(\mathbf{x})+\alpha \cdot\left(x_{\mathrm{c}}-\mathrm{FLP}(\mathbf{x})\right)
$$

is also realizable in $\mathrm{LA}_{\text {sqrt }}$ for dyadic sharpening gain $\alpha \geq 0$.

\section{$5 \quad$ Sharpening Filters Portfolio}

Various low-pass fuzzy filters with various but symmetric masks will be used for image sharpening with dyadic gain. Resulting bank of fuzzy filters is determined by filtering approaches and mask weights.

\subsection{Fuzzy Filtering Approaches}

Various fuzzy sharpening approaches were used for 2D image enhancement. List of fuzzy low-pass filters is included in Tab. 1. Image sharpening gain was studied for $\alpha \in\{k / 32: k=0, \ldots, 160\}$ for various images and noise types.

Table 1: Low-Pass Filter Portfolio

\begin{tabular}{lll}
\hline Index & Filter & Description \\
\hline 1 & M & Median \\
2 & A & Average \\
3 & H & Hodges-Lehmann \\
4 & O & Opening \\
5 & C & Closing \\
6 & OCM & Opening-Closing Mean \\
7 & OC & Opening followed by Closing \\
8 & CO & Closing followed by Opening \\
9 & OCCOM & OC and CO Mean \\
10 & CF & Constrained Filter \\
\hline
\end{tabular}

\subsection{Compact $3 \times 3$ Masks}

In this study we used five small compact masks as

$$
\begin{gathered}
\mathbf{M}_{1}=\left(\begin{array}{lll}
0 & 1 & 0 \\
1 & 1 & 1 \\
0 & 1 & 0
\end{array}\right), \mathbf{M}_{2}=\left(\begin{array}{lll}
0 & 1 & 0 \\
1 & 4 & 1 \\
0 & 1 & 0
\end{array}\right), \\
\mathbf{M}_{3}=\left(\begin{array}{lll}
1 & 1 & 1 \\
1 & 1 & 1 \\
1 & 1 & 1
\end{array}\right), \mathbf{M}_{4}=\left(\begin{array}{lll}
1 & 1 & 1 \\
1 & 8 & 1 \\
1 & 1 & 1
\end{array}\right), \mathbf{M}_{5}=\left(\begin{array}{lll}
1 & 2 & 1 \\
2 & 4 & 2 \\
1 & 2 & 1
\end{array}\right)
\end{gathered}
$$

but only $\mathbf{M}_{2}, \mathbf{M}_{4}, \mathbf{M}_{5}$ are applicable in average filter.

\subsection{Compact $5 \times 5$ Masks}

Seven large compact masks were also used as

$$
\begin{aligned}
& \mathbf{M}_{6}=\left(\begin{array}{lllll}
0 & 0 & 1 & 0 & 0 \\
0 & 1 & 1 & 1 & 0 \\
1 & 1 & 1 & 1 & 1 \\
0 & 1 & 1 & 1 & 0 \\
0 & 0 & 1 & 0 & 0
\end{array}\right), \mathbf{M}_{7}=\left(\begin{array}{lllll}
0 & 0 & 1 & 0 & 0 \\
0 & 1 & 1 & 1 & 0 \\
1 & 1 & 4 & 1 & 1 \\
0 & 1 & 1 & 1 & 0 \\
0 & 0 & 1 & 0 & 0
\end{array}\right), \\
& \mathbf{M}_{8}=\left(\begin{array}{lllll}
0 & 1 & 1 & 1 & 0 \\
1 & 1 & 1 & 1 & 1 \\
1 & 1 & 1 & 1 & 1 \\
1 & 1 & 1 & 1 & 1 \\
0 & 1 & 1 & 1 & 0
\end{array}\right), \mathbf{M}_{9}=\left(\begin{array}{ccccc}
0 & 1 & 1 & 1 & 0 \\
1 & 1 & 1 & 1 & 1 \\
1 & 1 & 12 & 1 & 1 \\
1 & 1 & 1 & 1 & 1 \\
0 & 1 & 1 & 1 & 0
\end{array}\right),
\end{aligned}
$$




$$
\begin{gathered}
\mathbf{M}_{10}=\left(\begin{array}{lllll}
1 & 1 & 1 & 1 & 1 \\
1 & 1 & 1 & 1 & 1 \\
1 & 1 & 1 & 1 & 1 \\
1 & 1 & 1 & 1 & 1 \\
1 & 1 & 1 & 1 & 1
\end{array}\right), \mathbf{M}_{11}=\left(\begin{array}{lllll}
1 & 1 & 1 & 1 & 1 \\
1 & 1 & 1 & 1 & 1 \\
1 & 1 & 8 & 1 & 1 \\
1 & 1 & 1 & 1 & 1 \\
1 & 1 & 1 & 1 & 1
\end{array}\right), \\
\mathbf{M}_{12}=\left(\begin{array}{ccccc}
1 & 4 & 6 & 4 & 1 \\
4 & 16 & 24 & 16 & 4 \\
6 & 24 & 36 & 24 & 6 \\
4 & 16 & 24 & 16 & 4 \\
1 & 4 & 6 & 4 & 1
\end{array}\right)
\end{gathered}
$$

but only $\mathbf{M}_{7}, \mathbf{M}_{9}, \mathbf{M}_{11}, \mathbf{M}_{12}$ are applicable in average filter.

\subsection{Ring Masks}

The dyadic property was also obtained by removing central points. Resulting ring masks are

$$
\begin{gathered}
\mathbf{M}_{13}=\left(\begin{array}{lll}
0 & 1 & 0 \\
1 & 0 & 1 \\
0 & 1 & 0
\end{array}\right), \mathbf{M}_{14}=\left(\begin{array}{lll}
1 & 1 & 1 \\
1 & 0 & 1 \\
1 & 1 & 1
\end{array}\right), \\
\mathbf{M}_{15}=\left(\begin{array}{lllll}
0 & 0 & 1 & 0 & 0 \\
0 & 1 & 0 & 1 & 0 \\
1 & 0 & 0 & 0 & 1 \\
0 & 1 & 0 & 1 & 0 \\
0 & 0 & 1 & 0 & 0
\end{array}\right), \mathbf{M}_{16}=\left(\begin{array}{lllll}
0 & 1 & 1 & 1 & 0 \\
1 & 1 & 0 & 1 & 1 \\
1 & 0 & 0 & 0 & 1 \\
1 & 1 & 0 & 1 & 1 \\
0 & 1 & 1 & 1 & 0
\end{array}\right) .
\end{gathered}
$$

\section{Optimal Sharpening in $\mathrm{LA}_{\mathrm{sqrt}}$}

Numerical experiments on two representative images were performed to obtain the best possible increasing of Signal to Noise Ratio (SNR) as generally recommended criterion. Pixel intensity of ideal image was normalized into interval $[0,1]$, first. The random noise was added and resulting image was constrained to original range [0, 1] as model of real image in the second step. Various types of fuzzy sharpening filters with various masks and sharpening gains were applied to real image to obtain a set of enhanced images.

The $S N R$ criterion was designed using the logarithmic scale as

$$
S N R=10 \log _{10}\left(\frac{P_{\text {signal }}}{P_{\text {noise }}}\right)
$$

where $P_{\text {signal }}, P_{\text {noise }}$ are average powers of the signal and additive noise. The quality of sharpening was studied as improvement $\triangle S N R=S N R-S N R_{0}$ in $\mathrm{dB}$ where $S N R_{0}, S N R$ reflect the image quality before and after the sharpening.

A slice of human brain 2D MRI ROI of size $40 \times 40$ was used in the first case together with additive Gaussian noise $\sigma=0.05$ as example of low-level noise. Results of sharpening are included in Tabs. 2,3 as optimal increasing of SNR value and adequate sharpening gain. The symbol * means that the filter cannot be realized in the fuzzy system. The best results were obtained for averaging filter $\mathrm{F}_{2}$ with four-point ring mask $\mathbf{M}_{13}$. The same mask also produced acceptable increasing of $\mathrm{SNR}$ for Hodges-Lehmann median $\mathrm{F}_{3}$, median $\mathrm{F}_{1}$, and constraind filter $\mathrm{F}_{10}$ with $\alpha=13 / 32$ in majority of cases. The open-closing filter $\mathrm{F}_{6}$ is also acceptable with five-point mask $\mathrm{M}_{1}$ and $\alpha=1 / 16$. The noised MRI image and the result of the best filter $\mathrm{F}_{2}$ are depicted in Fig. 1.

The same image of size $40 \times 40$ was used in the second case with spiky noise generated as follows. Additive Gaussian noise with $\sigma=0.1$ was corrupted by pepper-salt noise with probability 0.05 . Results of sharpening are included in Tabs. 4,5 as optimal SNR increasing values and adequate sharpening gains. The best results were obtained for Hodges-Lehmann median filter $\mathrm{F}_{3}$ with binomial mask $\mathbf{M}_{12}$ and $\alpha=0$. The median $\mathrm{F}_{1}$, OCCOM $F_{9}$, and constrained $\mathrm{F}_{10}$ filters over-performed the average filter $\mathrm{F}_{2}$ in $\mathrm{SNR}$ improvement. The same MRI image corrupted by spiky noise and the result of the best filter $\mathrm{F}_{3}$ are depicted in Fig. 1.

\section{Conclusions}

The fuzzy low-pass and sharpening filters have been investigated in the case of MRI images with Gaussian and spiky noise. The best results in $\triangle S N R$ were obtained for Hodges-Lehmann low-pass filter with binomial $5 \times 5$ 

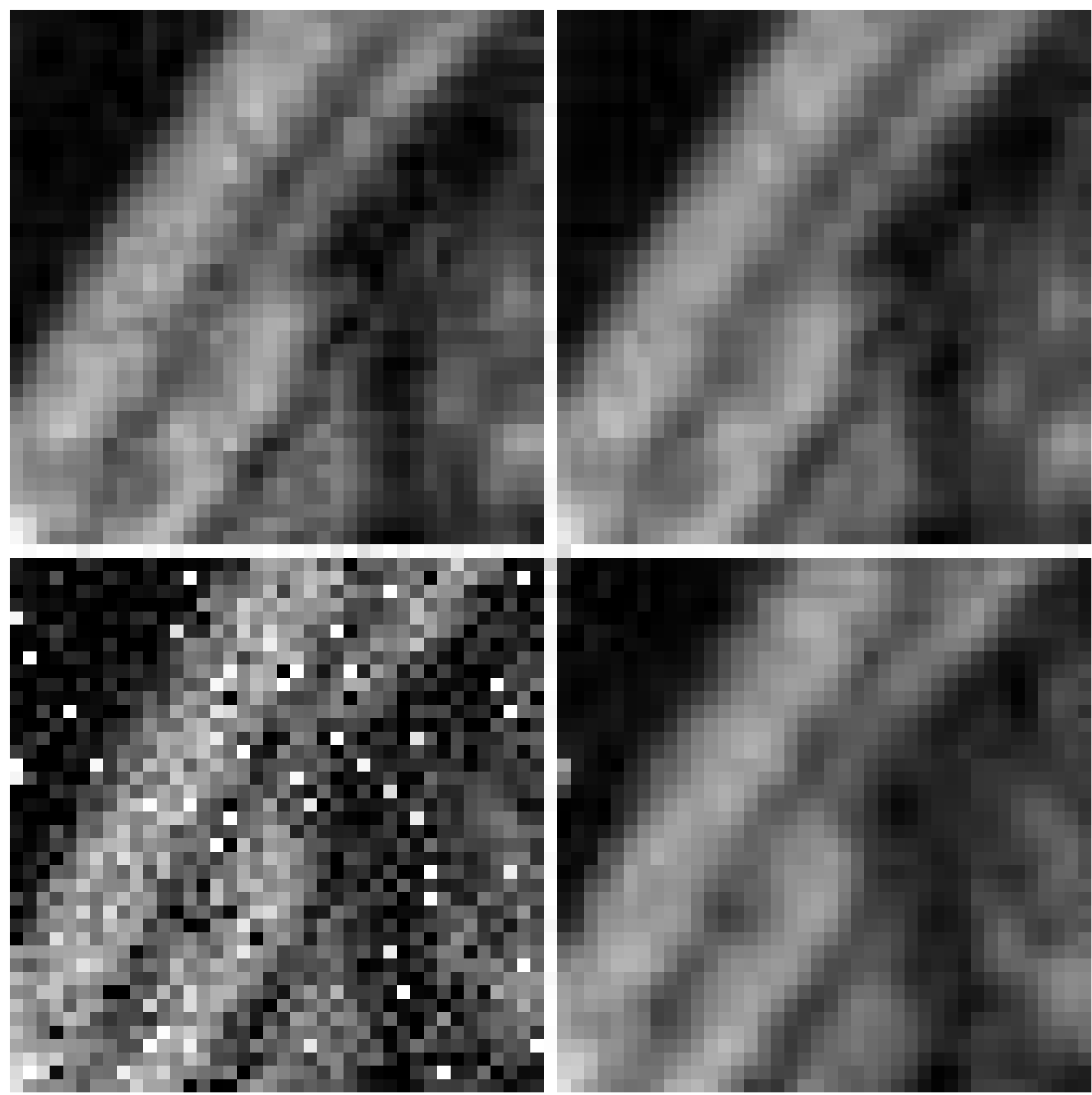

Figure 1: Performance of fuzzy filters: Gaussian-noised image (left top), smoothing by dyadic average (right top), spiky-noised image (left bottom), smoothing by Hodges-Lehmann median (bottom right)

mask in the case of pepper-salt noise. The same filtering approach was also suitable in the case of Gaussian noise however the best choice is dyadic average low-pass filter with four-point ring $3 \times 3$ mask in the Gaussian case. This study can be useful for future biomedical applications with unknown noise type and range when the Hodges-Lehmann low-pass filter with binomial $5 \times 5$ mask frequently overperforms traditional linear, median, and morphological filters and the sharpening is omitted.

Acknowledgement: This paper is supported by SGS17/196/OHK4/3T/14 grant of Czech Technical University in Prague, grant GA17-05840S of the Czech Science Foundation, and MSMT No 21-SVV/2018.

\section{References}

[1] Hajek, P.: Metamathematics of Fuzzy Logic. Kluwer, Dordrecht (1998)

[2] Novak, V., Perfilieva, I., Mockor, J.: Mathematical Principles of Fuzzy Logic. Kluwer Academic Publishers, Boston (1999)

[3] Gonzalez, R.C., Woods, R.E.: Digital Image Processing (3rd edition). Prentice Hall, New York (2008) 
Table 2: Sharpening quality as $\Delta S N R[\mathrm{~dB}]$ for Gaussian noise

\begin{tabular}{rcccccccccc}
\hline MASK & $\mathrm{F}_{1}$ & $\mathrm{~F}_{2}$ & $\mathrm{~F}_{3}$ & $\mathrm{~F}_{4}$ & $\mathrm{~F}_{5}$ & $\mathrm{~F}_{6}$ & $\mathrm{~F}_{7}$ & $\mathrm{~F}_{8}$ & $\mathrm{~F}_{9}$ & $\mathrm{~F}_{10}$ \\
\hline 1 & 1.37 & $*$ & 1.71 & 0.66 & 0.42 & 1.18 & 0.76 & 0.67 & 0.86 & $*$ \\
2 & 0.43 & 1.78 & 1.24 & 0.66 & 0.42 & 1.18 & 0.76 & 0.67 & 0.86 & 1.77 \\
3 & 1.51 & $*$ & 1.66 & 0.46 & 0.21 & 0.75 & 0.40 & 0.25 & 0.43 & $*$ \\
4 & 0.21 & 1.53 & 1.26 & 0.46 & 0.21 & 0.75 & 0.40 & 0.25 & 0.43 & 1.50 \\
5 & 1.35 & 1.69 & 1.73 & 0.46 & 0.21 & 0.75 & 0.40 & 0.25 & 0.43 & 1.68 \\
6 & 1.13 & $*$ & 1.07 & 0.29 & 0.13 & 0.47 & 0.23 & 0.14 & 0.24 & $*$ \\
7 & 0.89 & 0.94 & 1.12 & 0.29 & 0.13 & 0.47 & 0.23 & 0.14 & 0.24 & 0.94 \\
8 & 0.86 & $*$ & 0.67 & 0.18 & 0.09 & 0.33 & 0.14 & 0.08 & 0.15 & $*$ \\
9 & 0.39 & 0.54 & 0.77 & 0.18 & 0.09 & 0.33 & 0.14 & 0.08 & 0.15 & 0.55 \\
10 & 0.74 & $*$ & 0.54 & 0.13 & 0.03 & 0.19 & 0.09 & 0.03 & 0.08 & $*$ \\
11 & 0.55 & 0.41 & 0.62 & 0.13 & 0.03 & 0.19 & 0.09 & 0.03 & 0.08 & 0.41 \\
12 & 1.24 & 1.04 & 1.25 & 0.13 & 0.03 & 0.19 & 0.09 & 0.03 & 0.08 & 1.04 \\
13 & 1.67 & 1.85 & 1.79 & 0.54 & 0.31 & 0.90 & 0.57 & 0.48 & 0.61 & 1.85 \\
14 & 1.65 & 1.55 & 1.66 & 0.43 & 0.20 & 0.69 & 0.41 & 0.29 & 0.44 & 1.55 \\
15 & 0.81 & 0.58 & 0.63 & 0.21 & 0.09 & 0.09 & 0.18 & 0.12 & 0.19 & 0.58 \\
16 & 0.63 & 0.36 & 0.42 & 0.17 & 0.09 & 0.09 & 0.14 & 0.09 & 0.15 & 0.36 \\
\hline
\end{tabular}

Table 3: Sharpening gain $\alpha$ (in 1/32) for Gaussian noise

\begin{tabular}{rcccccccccc}
\hline MASK & $\mathrm{F}_{1}$ & $\mathrm{~F}_{2}$ & $\mathrm{~F}_{3}$ & $\mathrm{~F}_{4}$ & $\mathrm{~F}_{5}$ & $\mathrm{~F}_{6}$ & $\mathrm{~F}_{7}$ & $\mathrm{~F}_{8}$ & $\mathrm{~F}_{9}$ & $\mathrm{~F}_{10}$ \\
\hline 1 & 9 & $*$ & 8 & 16 & 19 & 2 & 17 & 18 & 14 & $*$ \\
2 & 0 & 0 & 0 & 16 & 19 & 2 & 17 & 18 & 14 & 0 \\
3 & 11 & $*$ & 11 & 22 & 25 & 13 & 23 & 25 & 22 & $*$ \\
4 & 0 & 0 & 0 & 22 & 25 & 13 & 23 & 25 & 22 & 1 \\
5 & 5 & 7 & 6 & 22 & 25 & 13 & 23 & 25 & 22 & 8 \\
6 & 15 & $*$ & 16 & 26 & 28 & 20 & 27 & 28 & 26 & $*$ \\
7 & 9 & 14 & 11 & 26 & 28 & 20 & 27 & 28 & 26 & 14 \\
8 & 19 & $*$ & 21 & 28 & 29 & 23 & 28 & 29 & 28 & $*$ \\
9 & 7 & 17 & 10 & 28 & 29 & 23 & 28 & 29 & 28 & 17 \\
10 & 20 & $*$ & 22 & 29 & 30 & 26 & 29 & 31 & 29 & $*$ \\
11 & 15 & 22 & 18 & 29 & 30 & 26 & 29 & 31 & 29 & 22 \\
12 & 12 & 16 & 13 & 29 & 30 & 26 & 29 & 31 & 29 & 16 \\
13 & 14 & 13 & 13 & 17 & 21 & 6 & 19 & 20 & 18 & 13 \\
14 & 14 & 14 & 14 & 22 & 25 & 14 & 23 & 24 & 21 & 14 \\
15 & 21 & 22 & 22 & 27 & 28 & 28 & 27 & 28 & 27 & 22 \\
16 & 22 & 25 & 24 & 28 & 29 & 29 & 28 & 29 & 28 & 25 \\
\hline
\end{tabular}

[4] Azevedo, F.: Constraint Solving over Multi-Valued Logics: Application to Digital Circuits. IOS Press, New York (2003)

[5] Majerova, D.: Image Processing by Means of Lukasiewicz Algebra with Square Root. PhD Thesis, ICT Prague (2004)

[6] Wasserman, S., Faust, K.: Social Network Analysis: Methods and Applications (2nd edition). Cambridge University Press, New York (2006)

[7] Arce, G.R.: Nonlinear Signal Processing: A Statistical Approach. Wiley, New Jersey (2005)

[8] Arias-Castro, E., Donoho, D.L.: Does Median Filtering Truly Preserve Edges Better than Linear Filtering. Annals of Statistics 37(3), 1172-1196 (2009)

[9] Hyndman, R.J., Fan, Y.: Sample Quantiles in Statistical Packages. American Statistician 50(4), 361-365 (1996)

[10] Hall, M.: Smooth Operator: Smoothing Seismic Horizons and Attributes. The Leading Edge 26(1), 16-20 (2007)

[11] Hodges, J.L., Lehmann, E.L.: Estimates of Location Based on Rank Tests. The Annals of Mathematical Statistics 34(2), 598-611 (1963)

[12] Mitra, S.K., Kaiser, J.F.: Handbook for Digital Signal Processing. John Wiley, New York (1993) 
Table 4: Sharpening quality as $\Delta S N R[\mathrm{~dB}]$ for spiky noise

\begin{tabular}{rcccccccccc}
\hline MASK & $\mathrm{F}_{1}$ & $\mathrm{~F}_{2}$ & $\mathrm{~F}_{3}$ & $\mathrm{~F}_{4}$ & $\mathrm{~F}_{5}$ & $\mathrm{~F}_{6}$ & $\mathrm{~F}_{7}$ & $\mathrm{~F}_{8}$ & $\mathrm{~F}_{9}$ & $\mathrm{~F}_{10}$ \\
\hline 1 & 6.67 & $*$ & 7.39 & 3.95 & 0.93 & 5.15 & 6.28 & 6.00 & 8.09 & $*$ \\
2 & 2.42 & 4.40 & 4.13 & 3.95 & 0.93 & 5.15 & 6.28 & 6.00 & 8.09 & 6.33 \\
3 & 8.38 & $*$ & 8.51 & 3.13 & 0.63 & 4.64 & 4.73 & 3.60 & 6.84 & $*$ \\
4 & 1.85 & 4.71 & 4.57 & 3.13 & 0.63 & 4.64 & 4.73 & 3.60 & 6.84 & 7.34 \\
5 & 7.08 & 6.66 & 8.08 & 3.13 & 0.63 & 4.64 & 4.73 & 3.60 & 6.84 & 7.37 \\
6 & 8.23 & $*$ & 8.26 & 2.88 & 0.61 & 4.67 & 4.14 & 3.31 & 6.36 & $*$ \\
7 & 7.48 & 6.18 & 7.76 & 2.88 & 0.61 & 4.67 & 4.14 & 3.31 & 6.36 & 7.32 \\
8 & 7.99 & $*$ & 7.93 & 2.09 & 0.43 & 3.55 & 2.96 & 2.23 & 5.34 & $*$ \\
9 & 5.13 & 5.60 & 5.42 & 2.09 & 0.43 & 3.55 & 2.96 & 2.23 & 5.34 & 7.07 \\
10 & 7.70 & $*$ & 7.60 & 1.71 & 0.37 & 3.15 & 2.26 & 1.79 & 4.56 & $*$ \\
11 & 7.36 & 6.19 & 7.03 & 1.71 & 0.37 & 3.15 & 2.26 & 1.79 & 4.56 & 6.95 \\
12 & 8.55 & 7.17 & 8.61 & 1.71 & 0.37 & 3.15 & 2.26 & 1.79 & 4.56 & 7.41 \\
13 & 7.21 & 5.80 & 7.25 & 3.74 & 0.73 & 4.54 & 6.07 & 5.75 & 7.37 & 5.80 \\
14 & 8.56 & 6.94 & 8.34 & 3.48 & 0.80 & 5.12 & 5.36 & 4.92 & 7.32 & 7.08 \\
15 & 7.24 & 5.95 & 7.16 & 3.23 & 1.02 & 5.01 & 4.83 & 4.16 & 6.25 & 6.04 \\
16 & 7.10 & 6.10 & 7.07 & 2.69 & 0.91 & 5.07 & 3.96 & 3.44 & 5.98 & 6.20 \\
\hline
\end{tabular}

Table 5: Sharpening gain $\alpha$ (in 1/32) for spiky noise

\begin{tabular}{rcccccccccc}
\hline MASK & $\mathrm{F}_{1}$ & $\mathrm{~F}_{2}$ & $\mathrm{~F}_{3}$ & $\mathrm{~F}_{4}$ & $\mathrm{~F}_{5}$ & $\mathrm{~F}_{6}$ & $\mathrm{~F}_{7}$ & $\mathrm{~F}_{8}$ & $\mathrm{~F}_{9}$ & $\mathrm{~F}_{10}$ \\
\hline 1 & 0 & $*$ & 0 & 6 & 11 & 0 & 4 & 2 & 0 & $*$ \\
2 & 0 & 0 & 0 & 6 & 11 & 0 & 4 & 2 & 0 & 0 \\
3 & 1 & $*$ & 1 & 11 & 20 & 0 & 8 & 9 & 2 & $*$ \\
4 & 0 & 0 & 0 & 11 & 20 & 0 & 8 & 9 & 2 & 0 \\
5 & 0 & 0 & 0 & 11 & 20 & 0 & 8 & 9 & 2 & 0 \\
6 & 2 & $*$ & 1 & 13 & 21 & 0 & 10 & 11 & 3 & $*$ \\
7 & 0 & 0 & 0 & 13 & 21 & 0 & 10 & 11 & 3 & 0 \\
8 & 2 & $*$ & 2 & 17 & 25 & 4 & 15 & 16 & 6 & $*$ \\
9 & 0 & 0 & 0 & 17 & 25 & 4 & 15 & 16 & 6 & 0 \\
10 & 3 & $*$ & 3 & 20 & 26 & 6 & 18 & 18 & 8 & $*$ \\
11 & 0 & 0 & 0 & 20 & 26 & 6 & 18 & 18 & 8 & 0 \\
12 & 0 & 0 & 0 & 20 & 26 & 6 & 18 & 18 & 8 & 0 \\
13 & 4 & 7 & 4 & 6 & 14 & 0 & 3 & 2 & 0 & 7 \\
14 & 3 & 5 & 3 & 9 & 16 & 0 & 6 & 5 & 1 & 5 \\
15 & 4 & 6 & 4 & 10 & 15 & 0 & 8 & 8 & 3 & 6 \\
16 & 4 & 5 & 4 & 14 & 19 & 0 & 11 & 11 & 5 & 5 \\
\hline
\end{tabular}

